\title{
Double poles in Lattice QCD with mixed actions
}

\author{
Maarten Golterman, ${ }^{a}$ Taku Izubuchi ${ }^{b}$ and Yigal Shamir $^{c}$ \\ ${ }^{a}$ Department of Physics and Astronomy, San Francisco State University \\ San Francisco, CA 94132, USA \\ maarten@stars.sf su . edu \\ ${ }^{b}$ RIKEN-BNL Research Center, Brookhaven National Laboratory \\ Upton, NY 11973, USA, and \\ Institute for Theoretical Physics, Kanazawa University \\ Kakuma, Kanazawa, 920-1192, Japan \\ izubuchi@quark.phy.bnl.gov \\ ${ }^{c}$ School of Physics and Astronomy \\ Raymond and Beverly Sackler Faculty of Exact Sciences \\ Tel-Aviv University, Ramat Aviv, 69978 ISRAEL \\ shamir@post.tau.ac.il
}

\begin{abstract}
We consider effects resulting from the use of different discretizations for the valence and the sea quarks, considering Wilson and/or Ginsparg-Wilson fermions. We assume that such effects appear through scaling violations that can be studied using effective-lagrangian techniques. We show that a double pole is present in flavor-neutral Goldstone meson propagators, even if the flavor non-diagonal Goldstone mesons made out of valence or sea quark have equal masses. We then consider some observables known to be anomalously sensitive to the presence of a double pole. We find that the double-pole enhanced scaling violations may turn out to be rather small in practice.
\end{abstract}




\section{Introduction}

The use of mixed actions in Lattice QCD (LQCD) is at present gaining popularity. With "mixed action" we refer to any simulation in which different lattice fermions are used in the valence and sea sectors. (If only the bare masses differ, we are dealing with a partially-quenched (PQ) theory 11.) The main reason is that the generation of dynamical-fermion configurations is very expensive, while for many quantities the "good quality" of symmetries (such as chiral and flavor or taste symmetries) is more important in the valence sector than in the sea sector. An example of this are the powerlike subtractions needed for $K \rightarrow \pi$, for which good chiral symmetry in the valence sector is essential, but not that in the sea sector.

However, the use of mixed actions raises field-theoretical questions. At non-vanishing lattice spacing $a$ a mixed-action theory is not unitary, and the question is what happens to unitarity in the continuum limit, as well as how big the effects of the violation of unitarity are at the lattice spacings used in actual simulations. We note that similar issues arise for many discretizations of LQCD which do not employ mixed actions, such as improved actions and actions with Ginsparg-Wilson (GW) fermions.

Here we address the second question, the size of effects at non-zero lattice spacing. We extend the notion of universality to assume that unphysical effects due to the use of a mixed action vanish in the continuum limit, and are controlled by positive powers of $a$. We further assume that effective-field theory (EFT) techniques can be used to investigate the behavior of mixedaction theories at low energy.

The most salient phenomenon which occurs if the valence quarks don't match the sea quarks is the appearance of a double pole in flavor-neutral Goldstone meson propagators, with a residue $R$ proportional to $a^{2}$, as will be explained below. If also the renormalized quark masses are not equal, there is also a contribution from the quark mass difference, $R \propto c_{1} a^{2}+$ $c_{2}\left(m_{\text {sea }}-m_{\text {valence }}\right)$ [1. Since the double pole represents a dramatic change of the infrared (IR) behavior of the theory, it is important to investigate the most serious consequences of the double pole. As an example of this, we consider the enhanced finite-volume effects in $I=0$ pion scattering. For a more detailed account of this work, see Ref. 2]. In our work, we deal only with the cases in which the fermions in both sectors are Wilson-like or GW 
fermions. ${ }^{1}$

\section{The double pole}

According to our assumptions, a mixed-action theory is a PQ theory in the continuum limit 2]. We therefore start with continuum chiral perturbation theory (ChPT) at lowest order, described by the PQ lagrangian [1]

$$
L_{\text {cont }}=\frac{1}{8} f^{2} \operatorname{str}\left(\partial_{\mu} \Sigma \partial_{\mu} \Sigma^{\dagger}\right)-\frac{1}{4} f^{2} B_{0} \operatorname{str}\left(\Sigma M^{\dagger}+M \Sigma^{\dagger}\right),
$$

where $\Sigma=\exp (2 i \Phi / f)$ is the non-linear meson field, $f$ and $B_{0}$ are the well-known lowest-order low-energy constants (LECs), and $M=$ $\operatorname{diag}\left(m_{v}, m_{v}, \ldots, m_{s}, m_{s}, \ldots, m_{v}, m_{v}, \ldots\right)$ the quark mass matrix, with $K$ valence quarks of mass $m_{v}, N$ sea quarks of mass $m_{s}$, and $K$ ghost quarks of mass $m_{v} \cdot{ }^{2}$ The relevant chiral symmetry group is thus $S U(K+N \mid K)_{L} \times$ $S U(K+N \mid K)_{R}$.

The next step we need is provided by the systematic analysis of Ref. 4, where mixed actions with a Wilson sea and GW valence quarks were considered in ChPT up to order $a^{2}$. It is very easy to generalize that work to the slightly more general case where the valence and sea sectors can be any combination of Wilson-like and GW fermions. ${ }^{3}$

The first observation is that at $a \neq 0$ the symmetry group is smaller. For GW valence quarks and a Wilson-like sea the symmetry group is

$$
\left[S U(K \mid K)_{L} \times S U(K \mid K)_{R}\right] \times S U(N) \quad(\mathrm{GW}-\text { Wilson }),
$$

while the case of Wilson-like valence and sea quarks (but with unequal lattice actions) the symmetry group is even smaller:

$$
S U(K \mid K) \times S U(N) \quad(\text { Wilson }- \text { Wilson }),
$$

\footnotetext{
${ }^{1}$ For mixed actions with staggered sea, see Ref. 3 .

${ }^{2}$ We take degenerate valence and degenerate sea quarks, but it is straightforward to generalize.

${ }^{3}$ With "Wilson-like" we refer to any type of Wilson fermion, including for instance clover and twisted-mass Wilson fermions. Our conclusions can be straightforwardly generalized to these situations. With GW fermions we refer to overlap fermions or domain-wall fermions with a small enough residual mass. A potentially interesting special case is the combination of untwisted Wilson sea quarks and twisted Wilson valence quarks. If this is done by only twisting the mass matrix of the valence quarks, this is a PQ theory, and not a (genuinely) mixed theory. The relevant symmetry group in that case is $S U(K+N \mid K)$.
} 
because all chiral symmetries are now broken.

Up to order $a^{2}$ there are a number of new operators which appear in the effective lagrangian, and we only give the ones here which are relevant for our investigation,

$$
\begin{gathered}
\delta L_{W}=-\frac{(a f)^{2}}{32}\left(\gamma_{v v}\left(\operatorname{str}\left(P_{v}\left(\Sigma-\Sigma^{\dagger}\right)\right)\right)^{2}+\gamma_{s s}\left(\operatorname{str}\left(P_{s}\left(\Sigma-\Sigma^{\dagger}\right)\right)\right)^{2}\right. \\
\left.+\gamma_{v s} \operatorname{str}\left(P_{v}\left(\Sigma-\Sigma^{\dagger}\right)\right) \operatorname{str}\left(P_{s}\left(\Sigma-\Sigma^{\dagger}\right)\right)\right)
\end{gathered}
$$

in which $\gamma_{v v, v s, s s}$ are new LECs, and $P_{v, s}$ are projectors on the valence and sea sectors, respectively. We note that $\gamma_{v v}=\gamma_{v s}=0$ when the valence quarks are $\mathrm{GW}$, because these operators break chiral symmetry, and thus are excluded by the symmetry group (2), but not by the group (3).

In order to obtain the meson propagators, we need to expand the lagrangian to quadratic order in the field $\Phi$. If we integrate out the sea- $\eta^{\prime}$ (which is heavy because of the singlet part of the $\eta^{\prime}$ mass), we have that $\operatorname{str}(\Phi)=\operatorname{str}\left(\left(P_{v}+P_{s}\right) \Phi\right)=0$, and Eq. (44) reduces to quadratic order to

$$
\delta L_{W}=\frac{1}{2} a^{2}\left(\gamma_{v v}+\gamma_{s s}-2 \gamma_{v s}\right)\left(\operatorname{str}\left(P_{v} \Phi\right)\right)^{2}+\ldots .
$$

Recognizing that $\operatorname{str}\left(P_{v} \Phi\right)$ is nothing else than the "super- $\eta$ " field of quenched QCD, it is obvious that $\delta L_{W}$ leads to a double pole in flavor-neutral propagators.

In the flavor non-diagonal sector, we find (tree-level) Goldstone meson masses [4]

$$
\begin{aligned}
& M_{v v}^{2}=2 B_{0 v} m_{v}+2 W_{0 v} a+2 \beta_{v} a^{2}, \\
& M_{s s}^{2}=2 B_{0 s} m_{s}+2 W_{0 s} a+2 \beta_{s} a^{2},
\end{aligned}
$$

where we now allowed for the fact that with the reduced symmetry groups the LECs $B_{0}, W_{0}$ and $\beta$ can be different in the valence and sea sectors. These equations tell us (to leading order in ChPT) what it means to set renormalized valence and sea quark masses equal: one chooses $m_{v, s}$ such that $M_{v v}=M_{s s}$. We note that $W_{0 v, s}=0$ if order- $a$ improved Wilson fermions are used, and that $W_{0 v, s}=0$ as well as $\beta_{v, s}=0$ for GW fermions.

Flavor-neutral propagators are given by ( $i$ and $j$ are flavor indices)

$$
\left\langle\Phi_{i i} \Phi_{j j}\right\rangle(p)=\left(\delta_{i j}-\frac{1}{N}\right) \frac{1}{p^{2}+M_{v v}^{2}}-\frac{R}{\left(p^{2}+M_{v v}^{2}\right)^{2}},
$$


with

$$
R=\frac{1}{N}\left(M_{s s}^{2}-M_{v v}^{2}\right)+\left(\gamma_{v v}+\gamma_{s s}-2 \gamma_{v s}\right) a^{2} .
$$

It is clear that $R \neq 0$ even if $M_{v v}=M_{s s}$. In fact, one may either choose the valence quark mass such that $R=0$, or choose it such that $M_{v v}=M_{s s}$ and live with a non-vanishing $R$. Either way, the appearance of a double pole due to scaling violations in mixed actions is relevant for hadronic quantities sensitive to the double pole, especially if the effects are enhanced because of the IR-singular nature of the double pole.

\section{Example: $I=0$ pion scattering}

It is easy to find quantities particularly sensitive to the double pole. Examples are the $I=0$ two-pion energy in a finite volume (which are related to the corresponding phase shifts) [5], the anomalous large-time behavior of the $a_{0}$ propagator [6], and the anomalous large-distance behavior of the nucleonnucleon potential [7]. Here we will just briefly consider the finite-volume two-pion energy; for the $a_{0}$ we refer to Ref. [2].

The energy of two pions at rest in an $I=0$ state, and in a finite spatial box of linear dimension $L$ with periodic boundary conditions, is given by (to one loop) [5, 2]

$$
\frac{\Delta E_{I=0}}{2 M_{v v}}=-\frac{7 \pi}{8 f^{2} M_{v v} L^{3}}+\frac{1}{2} B_{0}\left(M_{v v} L\right) \delta^{2}+\frac{1}{2}\left(1-\frac{1}{N}\right) A_{0}\left(M_{v v} L\right) \delta \epsilon+O\left(\epsilon^{2}\right),
$$

where

$$
\begin{aligned}
\delta & =\frac{R}{8 \pi^{2} f^{2}}, \quad \epsilon=\frac{M_{v v}^{2}}{16 \pi^{2} f^{2}} \\
B_{0}(M L) & =-0.53+O\left(1 /(M L)^{2}\right) \\
A_{0}(M L) & =49.59 /(M L)^{2}+O\left(1 /(M L)^{3}\right) .
\end{aligned}
$$

Setting $M_{v v}=M_{s s} \equiv M$, we may consider two different regimes, one in which $\epsilon \sim M^{2} / \Lambda^{2} \sim a \Lambda_{Q C D}$ [4], and one in which $\epsilon \sim M^{2} / \Lambda^{2} \sim\left(a \Lambda_{Q C D}\right)^{2}$ [8], where $\Lambda \sim 1 \mathrm{GeV}$ is the chiral symmetry breaking scale. In the first of these two regimes, the ratio of the one-loop to the tree-level terms in Eq. (9) is of order $\epsilon^{3} \times(M L)^{3}$ and $\epsilon^{2} \times M L$ for the $\delta^{2}$, respectively, $\delta \epsilon$ terms. In the second regime this ratio is of order $\epsilon \times(M L)^{3}$. 
One notes that the one-loop contributions are suppressed by positive powers of $\epsilon$ in both regimes, but for the positive powers of $M L$ which accompany these powers of $\epsilon$. This is an example of the enhancement due to the "sick" IR behavior of the double pole - these terms would all disappear when $R=0$. But in a mixed-action theory at $a \neq 0, R \neq 0$ even at $M_{v v}=M_{s s}$.

To get an idea about the size of these effects, one may substitute some typical values for the parameters of a LQCD simulation. Choosing for example $a \Lambda_{Q C D} \sim 0.1, a M \sim 0.2$ and $L / a \sim 32$, one finds that the $\delta$ terms in Eq. (9) represent scaling violations of order $10 \%$. This is small, but not negligible. Of course, in this estimate, we took all other constants (including the coefficient of $a^{2}$ in $R$ ) equal to one. Actual simulations are needed to make further progress with the investigation of the size of these effects.

\section{Conclusion}

Let us summarize what we learned about the use of mixed actions for LQCD computations.

We begin by re-emphasizing that we assumed that the unphysical effects of mixed actions are encoded in scaling violations, and that EFT techniques can be used to study the issue. We recall that this approach has proven very successful in similar cases, such as the unphysical effects due to (partial) quenching. It is therefore important to test this assumption also in this case through actual simulations, in which in addition the numerical size of the effects can be estimated more reliably than through our parametric estimates above and in Ref. [2].

We have concentrated on the role of the double pole, because it is the most IR-sensitive probe of unphysical effects due to the use of mixed actions, just as it is in the case of (partial) quenching. Clearly, the effects are quantity dependent, and are expected to be most pronounced for those quantities for which there is a clear unphysical enhancement. The example we gave is that of enhanced finite-volume effects in pion scattering. We concluded in that case that the effects are likely to be rather small numerically in present-day simulations, but not so small as to be automatically negligible.

Finally, we observe that in the case of the $I=0$ two-pion energy, this effect can also be monitored if one leaves out the "double-annihilation" diagram (as was done in Ref. 9] because of poor statistics), because the effective theory can be adapted to reflect this situation [5]. For the terms shown in 
Eq. (9) this corresponds to dropping the $\delta^{2}$ term. Our $10 \%$ estimate above came largely from the $\delta \epsilon$ term, and it is thus plausible that the size of the effect does not become much smaller without the double-annihilation diagram.

\section{Acknowledgments}

We thank Oliver Bär and Steve Sharpe, and in particular Paulo Bedaque and Claude Bernard, for helpful discussions. This work was started during a visit of two of us (MG and YS) to Brookhaven National Lab and Columbia University, and we thank the RBC collaboration for discussions and hospitality. MG also thanks the Lawrence Berkeley National Lab Nuclear Theory Group for hospitality. MG is supported in part by the US Department of Energy, and YS is supported by the Israel Science Foundation under grant 222/02-1.

\section{References}

[1] C. W. Bernard and M. F. L. Golterman, Partially quenched gauge theories and an application to staggered fermions, Phys. Rev. D 49, 486 (1994) arXiv:hep-lat/9306005.

[2] M. Golterman, T. Izubuchi and Y. Shamir, The role of the double pole in lattice QCD with mixed actions, Phys. Rev. D 71, 114508 (2005) arXiv:hep-lat/0504013.

[3] O. Bär, C. Bernard, G. Rupak and N. Shoresh, Chiral perturbation theory for staggered sea quarks and Ginsparg-Wilson valence quarks,' arXiv:hep-lat/0503009.

[4] O. Bär, G. Rupak and N. Shoresh, Chiral perturbation theory for lattice QCD at O(alpha**2), Nucl. Phys. Proc. Suppl. 129, 185 (2004) arXiv:hep-lat/0309033.

[5] C. Bernard and M. Golterman, Finite volume two pion energies and scattering in the quenched approximation, Phys. Rev. D 53, 476 (1996) arXiv:hep-lat/9507004.

[6] W. A. Bardeen, A. Duncan, E. Eichten, N. Isgur and H. Thacker, Chiral loops and ghost states in the quenched scalar propagator, Phys. Rev. D 65, 014509 (2002) arXiv:hep-lat/0106008. 
[7] S. R. Beane and M. J. Savage, Partially-quenched nucleon nucleon scattering, Phys. Rev. D 67, 054502 (2003) arXiv:hep-lat/0210046.

[8] S. Aoki, Chiral perturbation theory with Wilson-type fermions including $a^{* * 2}$ effects: $N(f)=2$ degenerate case, Phys. Rev. D 68, 054508 (2003) arXiv:hep-lat/0306027.

[9] Y. Kuramashi, M. Fukugita, H. Mino, M. Okawa and A. Ukawa, Lattice QCD calculation of full pion scattering lengths, Phys. Rev. Lett. 71, 2387 (1993). 